\title{
LOUIS APPLEBY
}

\section{What about the workforce?}

How many consultants do we need nationally to deliver mental health care? It is a simple question, but it has no simple answer - except that we need substantially more than we have at the moment.

Of course, there are 'norms' - one consultant for every so-many people - but norms have been discredited over the years because they are not based on an agreed formula, and at times they look like nothing more than a forlorn hope. Another way of answering the question is by pointing to the number of vacant consultant posts - at least $12 \%$, according to the best available information. But some trusts do not create posts that they do not expect to fill, so the number of vacancies underestimates need. Then there is the number of posts occupied by locums, but if this tells us anything, it is about the

stability of mental health teams and the consistency of care that they provide.

A different approach to planning the psychiatric worforce is needed. We need to plan the number of doctors in mental health by tracking trainee figures from medical students through to consultants and beyond, into retirement. We need to answer several key questions.

For example, what is the variation between medical schools in the proportion of students choosing psychiatry, and how can it be explained? Why do many senior house officers (SHOs) leave psychiatry before completing their training? How many associate specialists are there in mental health and what are their prospects for training as consultants? How many specialist registrar $(\mathrm{SpR})$ posts can we realistically fill and where in the country should they be? How big is the problem of early retirement and what will persuade experienced colleagues to stay?

A new working party, convened jointly by the Department of Health and the College, is considering these and other matters. The aim is to produce an adequate and sustained increase in the number of psychiatrists working in adult mental health in England from the current figure of around 1600 whole time equivalents. The recent announcement of $135 \mathrm{SpR}$ posts will push this figure to over 2000 in the next 5 years, but this is just the start. We need models for workforce development that show in precise terms how increases in recruitment and retention of psychiatrists will carry through into the consultant workforce, both in the shortand long-term. This is a new and improved version of the numbers game. With the help of colleagues from the Medical Workforce Review Team (previously SWAG Specialty Workforce Advisory Group), and the right computer software, we can examine the potential impact on consultant numbers of a realistic expansion of people in training grades.

The Department of Health has already funded the College to carry out a study of SHO 'attrition' and the new working party will monitor this work. What it tells us about the experience of junior psychiatrists may not be comfortable to hear, but it should highlight the problems that we need to address if the current shortfall in consultants is going to be addressed within the next few years.

There, however, lies the biggest problem for workforce plans. Consultant psychiatrists are not created overnight - most solutions are long-term. So we also have to make a major effort to bring in doctors from other developed countries - although not the already under-doctored countries of the developing world. Of course, there are issues to tackle in doing this. We need to ensure satisfactory training, experience and language. We need to provide proper peer support. But there is now an NHS-wide initiative on international recruitment, and we need to be part of it.

Professor Louis Appleby National Director for Mental Health 